\title{
Estudo cariotípico de duas espécies brasileiras do gênero Micrurus (Ophidia: Elapidae)
}

\author{
Herbert Serafim ${ }^{1,2,3}$, Denise Maria Peccinini-Seale ${ }^{1}$ \& Radenka Francisca Batistic ${ }^{2}$ \\ Biota Neotropica v7 (n1) - http://www.biotaneotropica.org.br/v7n1/pt/abstract?article+bn01607012007 \\ Recebido em 13/06/06 \\ Versão reformulada recebida em 20/11/06 \\ Publicado em 15/01/07 \\ ${ }^{1}$ Laboratório de Genética de Répteis e Artrópodes, Departamento de Genética e Biologia Evolutiva, \\ Instituto de Biociências, Universidade de São Paulo, Rua do Matão, 277, CEP 05508-900, \\ Cid. Universitária, São Paulo, SP, Brasil \\ ${ }^{2}$ Laboratório de Herpetologia, Instituto Butantan, Avenida Vital Brasil, 1500, \\ CEP 05503-900, São Paulo, SP, Brasil \\ ${ }^{3}$ Autor para correspondência: Herbert Serafim,e-mail: herbert_ibusp@yahoo.com.br
}

\begin{abstract}
Serafim, H., Peccinini-Seale, D.M. \& Batistic, R.F. Karyotypic studies of two Brazilian species of the genus Micrurus (Ophidia: Elapidae). Biota Neotrop. Jan/Apr 2007 vol. 7, no. 1 http://www.biotaneotropica. org.br/v7n1/pt/abstract?article+bn01607012007 ISSN 1676-0603.

The karyotypes of two brazilian species of coral snakes (Ophidia: Elapidae): Micrurus corallinus and M. ibiboboca are described for the first time. Cytological preparations were obtained through the technique of squashing, followed by conventional Giemsa staining. The diploid number for Micrurus corallinus was 40 chromosomes with a karyotype formulae $40(4 ; 16 ; 20)$ with a secondary constriction in the second pair of subtelocentric macrochromosomes. Micrurus ibiboboca is described by the formulae $42(2 ; 20 ; 20)$ with $2 \mathrm{n}=42$ with a secondary constriction in the first pair of telocentric macrochromosomes. The karyotypes of M. corallinus and $M$. ibiboboca present aspects similar to M. lemniscatus and M. surinamensis, the only previously analyzed species of this genus in South America. These four species have the same number of microchromosomes (20), the largest number of telocentric or subtelocentric macrochromosomes and the largest diploid numbers (2n) ever found in the genus Micrurus. These characteristics differ from those of the nine known species of Central America.

Keywords: karyotype, Elapidae, cytogenetics, Micrurus, snakes.

\section{Resumo}

Serafim, H., Peccinini-Seale, D.M. \& Batistic, R.F. Estudo cariotípico de duas espécies brasileiras do gênero Micrurus (Ophidia: Elapidae). Biota Neotrop. Jan/Apr 2007 vol. 7, no. 1 http://www.biotaneotropica. org.br/v7n1/pt/abstract?article+bn01607012007 ISSN 1676-0603.

Descrevemos neste trabalho o cariótipo de duas espécies de cobra coral (Ophidia: Elapidae) do Brasil: Micrurus corallinus e M. ibiboboca. Preparações citológicas foram obtidas através da técnica de esmagamento, seguida de coloração convencional com Giemsa. Encontramos para M. corallinus, um número diplóide (2n) de 40 cromossomos, com fórmula cariotípica $40(4 ; 16 ; 20)$ e constricção secundária no segundo par de macrocromossomos subtelocêntricos. Micrurus ibiboboca apresentou $2 \mathrm{n}=42$, com fórmula cariotípica 42 $(2 ; 20 ; 20)$ e constricção secundária no primeiro par de macrocromossomos telocêntricos. As primeiras descrições dos cariótipos das espécies $M$. corallinus e M. ibiboboca, apresentadas neste trabalho, mostram aspectos semelhantes aos cariótipos de M. lemniscatus e M. surinamensis, únicas espécies deste gênero da América do Sul analisadas cromossomicamente. As quatro espécies em questão apresentam o mesmo número de microcromossomos (20), os maiores valores de macrocromossomos telocêntricos ou subtelocêntricos e os maiores números diplóides (2n) já encontrados no gênero Micrurus. Tais características diferenciam estas espécies da América do Sul das nove da América Central com cariótipos já estudados.
\end{abstract}

Palavras-chave: cariótipo, Elapidae, citogenética, Micrurus, serpentes. 


\section{Introdução}

Nas Américas, a Família Elapidae é representada pelas cobras corais. As 61 espécies reconhecidas estão divididas em três gêneros: Micrurus com 57 espécies, Leptomicrurus com três espécies e Micruroides com uma espécie. O gênero Micrurus ocorre desde a Argentina até o sul dos Estados Unidos (Melgarejo 2003). Na fauna brasileira há 22 espécies da Família Elapidae, a maioria pertencente ao gênero Micrurus (SBH 2006). No gênero Micrurus há dois grandes grupos distintos, tanto pelo padrão de distribuição dos anéis pretos (em tríades ou simples), quanto por algumas características morfológicas, como a forma dos hemipênis (curtos e apenas bilobados nas de tríades, longos e bifurcados nas de anéis simples); o grupo de tríades predomina nas áreas mais povoadas do Brasil, onde apenas $M$. corallinus representa as espécies de anel simples, enquanto na Amazônia ambos os padrões estão representados por diversas espécies (Melgarejo 2003). O estudo das relações evolutivas das espécies da família Elapidae tem abordado aspectos morfológicos (Savage \& Vial 1973, Roze 1982, 1996, Slowinski 1995), enzimáticos (Cadle \& Sarich, 1981, Slowinski 1995, Silva \& Sites 2001) e moleculares (Silva \& Sites 2001). As cobras corais das Américas são morfológica e ecologicamente conservadas (Cadle \& Sarich 1981), porém, são significantemente diferentes cariológica e bioquimicamente (Gutiérrez et al. 1988). No gênero Micrurus o número diplóide de cromossomos (2n) varia consideravelmente de espécie para espécie e mesmo entre subespécies de uma mesma espécie. Das 14 espécies que ocorrem na América Central, oito têm cariótipos descritos (Bolaños 1982). Destas, $M$ diastema apresenta duas subespécies com cariótipos descritos, sem diferença entre os cariótipos e $M$. nigrocinctus tem duas subespécies, com diferenças no número e morfologia dos cromossomos (Gutiérrez \& Bolaños, 1979, Gutiérrez \& Bolaños 1981; Gutiérrez et al. 1988; Luykx et al. 1992). Micrurus nigrocinctus nigrocinctus (Gutiérrez \& Bolaños, 1979), M. ruatanus (Luikx et al. 1992) e M. browni (Gutiérrez et al 1988) têm o mais baixo número diplóide, $2 \mathrm{n}=26$, enquanto M. lemniscatus (Beçak \& Beçak 1969), tem o mais alto, $2 \mathrm{n}=42$ cromossomos. As características cariotípicas apresentadas acima indicam a utilidade do estudo cromossômico para a sistemática e para a definição de espécies, principalmente quando características morfológicas não são suficientes para o esclarecimentos de problemas taxonômicos (Roze 1996). As fêmeas do gênero Micrurus apresentam um par de cromossomos sexuais heteromórficos do tipo ZW, enquanto os machos são homomórficos, ZZ, como em M. fulvius tenere (Graham 1977), M. diastema (Gutiérrez et al. 1988) e duas subespécies de M. nigrocinctus (Gutiérrez \& Bolaños 1979). Na família Elapidae o cromossomo sexual Z representa entre 10 e $12 \%$ do complemento cromossômico haplóide (Gutiérrez et al. 1988). No gênero Micrurus, todas as espécies com cariótipo estudado, apresentam uma constrição secundária em um par de macrocromossomos autossômicos. Segundo Gutiérrez \& Bolaños (1988), há necessidade de estudos de um número maior de espécies de corais, sendo também indispensável aplicar técnicas de bandamento cromossômico e de caracterização de DNA. Estes estudos são essenciais para melhor compreensão da evolução do genoma e esclarecimento do papel dos rearranjos cromossômicos no processo evolutivo. Das 11 espécies estudadas cariotipicamente nas Américas, apenas M. lemniscatus e
M. surinamensis ocorrem no Brasil. Micrurus lemniscatus é a única espécie com o cariótipo descrito no Brasil (Beçak \& Beçak 1969). Descrevemos neste trabalho o cariótipo de outras duas espécies brasileiras: $M$. corallinus e $M$. ibiboboca.

\section{Material e Métodos}

As informações referentes ao número de registro, sexo e procedência dos exemplares analisados neste estudo encontram-se na Tabela 1. O exemplar de M. corallinus utilizado neste trabalho foi coletado no estado de São Paulo, município de Iguape (24 38' 655" S e 47 28' 997" W, 28 m) (licença IBAMA-RAN. Proc. 02027.004018/01-26) e o de M. ibiboboca foi doado por fornecedor ao Instituto Butantan. Os exemplares estão depositados na coleção Alphonse Richard Hoge do Instituto Butantan, São Paulo, SP. Preparações citológicas foram obtidas pela técnica de esmagamento de fragmentos de órgãos. Foi administrada por via intraperitoneal, solução aquosa de colchicina a $1 \%$, na proporção de $0,1 \mathrm{~mL}$ para cada $100 \mathrm{~g}$ de massa do animal. Após 6 a 17 horas, sacrificou-se cada animal e extraiu-se o baço, fígado, intestino e testículos. Fragmentos dos órgãos foram colocados em solução hipotônica de $\mathrm{KCl}$ 0,075 M, por $30 \mathrm{~min}$, e em seguida, fixados em metanol/acido acético glacial, 3:1. Antes do esmagamento, fragmentos foram colocados em ácido acético $50 \%$ por 15 minutos e, em seguida, esmagados entre lâmina e lamínula. As lamínulas foram retiradas após imersão em álcool absoluto e gelo seco. As lâminas foram deixadas secar ao ar. Antes da coloração, as lâminas foram hidrolisadas com $\mathrm{HCl} 1 \mathrm{~N}$, por 10 minutos, em seguida lavadas em água corrente e coloração por 10 minutos com Giemsa (Merck) a 2\% em tampão fosfato $\mathrm{pH}=6,8$. Estabeleceu-se o número diplóide para cada exemplar após a análise de 40 metáfases, sendo que as melhores foram fotografadas em microscópio óptico Zeiss Axioskop 2 Plus e dispositivo fotográfico digital Nikon Coolpix 995R acoplado. Os cromossomos foram organizados aos pares, em ordem decrescente de tamanho. Para a classificação morfológica foi utilizada a nomenclatura adotada por Levan et al. (1964). Foi determinado o número fundamental (NF) de cada espécie, ou seja, a soma do número de braços dos macrocromossomos, considerando telocêntricos e subtelocêntricos como 1, metacêntricos e submetacêntricos como 2 braços (Matthey 1949). Os cariótipos foram descritos de acordo com a fórmula 2 n (I; II; III), sendo que o I se refere ao número de macrocromossomos metacêntricos e submetacêntricos,; o II, ao número de macrocromossomos telocêntricos e subtelocêntricos e o III, ao número de microcromossomos segundo Peccinini-Seale (1981).

\section{Resultados}

Os resultados são mostrados na Tabela 2 e nas Figuras 3 e 4. Micrurus corallinus (Figura 1) apresentou $2 \mathrm{n}=40$ e fórmula cariotípica $40(4 ; 16 ; 20)$. Entre os macrocromossomos, os pares 1 e 5 são submetacêntricos, os pares 7 e 9 são telocêntricos e os pares 2, 3, 4, 6, 8 e 10 são subtelocêntricos (Figura 3). Os microcromossomos são na maioria telocêntricos ou subtelocêntricos. Há uma constrição secundária no segundo par de macrocromossomos subtelocêntricos (Figura 3).

Tabela 1. Relação das espécies, números de protocolos, sexo e procedência dos espécimes estudados.

Table 1. Species, register numbers, sex and localities

\begin{tabular}{ccc}
\hline Espécie & $\begin{array}{c}\mathbf{N}^{\mathbf{0}} \text { de registro no } \\
\text { Instituto Butantan }\end{array}$ & Sexo \\
\hline Micrurus corallinus & IB - 71604 & Macho \\
Micrurus ibiboboca & IB - 70114 & Macho \\
\hline
\end{tabular}


Tabela 2. Revisão dos cariótipos de Micrurus (incluindo os dados deste trabalho).

Table 2. Review of karyotypes of the genus Micrurus including karyotypes of this study.

\begin{tabular}{|c|c|c|c|c|}
\hline Espécie & 2n (I, II, III) & $\begin{array}{c}\text { Posição e Morfologia do par c/ } \\
\text { Constricção secundária }\end{array}$ & $\begin{array}{l}\text { Padrão de } \\
\text { coloração }\end{array}$ & Autores \\
\hline M. alleni & $34(14,6,14)$ & $1^{\circ}$ par $\mathrm{T}$ & Mônade & Gutiérrez \& Bolaños (1979) \\
\hline M. browni & $26(10,6,10)$ & $1^{\circ}$ par SM & Mônade & Gutiérrez et al. (1988) \\
\hline M. corallinus & $40(4,16,20)$ & $2^{\circ}$ par ST & Mônade & Presente trabalho \\
\hline M. diastema apiatus & $30(12,2,16)$ & $1^{\circ}$ par SM & Mônade & Gutiérrez et al. (1988) \\
\hline M. diastema sapperi & $30(12,2,16)$ & $1^{\circ}$ par SM & Mônade & Gutiérrez et al. (1988) \\
\hline M. elegans & $30(10,6,14)$ & $1^{\circ}$ par SM & Mônade & Gutiérrez et al. (1988) \\
\hline M. fulvius & $32(16,16)$ & $1^{\circ}$ par SM & Mônade & Graham (1977) \\
\hline M. hippocrepis & $30(12,2,16)$ & $1^{\circ}$ par SM & Mônade & Gutiérrez et al. (1988) \\
\hline M. ibiboboca & $42(2,20,20)$ & $1^{\circ}$ par $\mathrm{T}$ & Tríade & Presente trabalho \\
\hline M. lemniscatus carvalhoi & $42(2,20,20)$ & $1^{\circ}$ par $\mathrm{T}$ & Tríade & Becak \& Becak (1969) \\
\hline M. multifasciatus & $34(12,2,20)$ & $2^{\circ}$ par SM & Bicolor & Gutiérrez \& Bolaños (1979) \\
\hline M. nigrocinctus mosquitensis & $30(12,4,14)$ & $1^{\circ}$ par SM & Mônade & Gutiérrez \& Bolaños (1979) \\
\hline M. nigrocinctus nigrocinctus & $26(10,6,10)$ & $1^{\circ}$ par SM & Mônade & Gutiérrez \& Bolaños (1979) \\
\hline M. ruatanus & $26(10,6,10)$ & $1^{\circ}$ par SM & Mônade & Luykx et al. (1992) \\
\hline M. surinamensis surinamensis & $38(6,12,20)$ & $2^{\circ}$ par SM & Tríade & Gutiérrez et al. (1988) \\
\hline
\end{tabular}

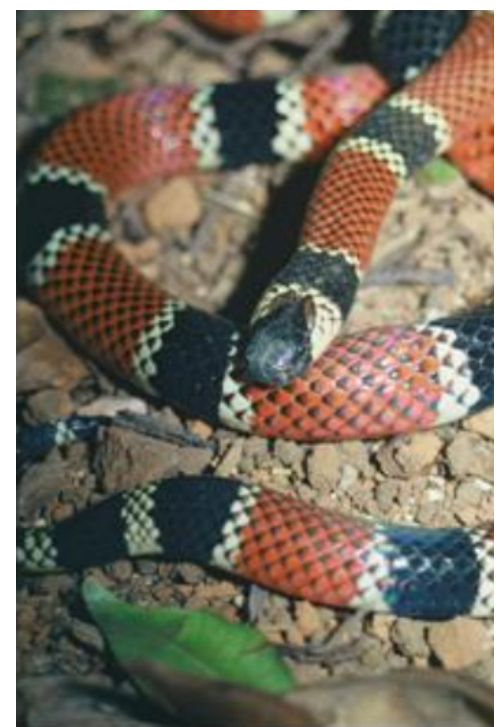

Figura 1. Micrurus corallinus (foto M. R. Duarte).

Figure 1. Micrurus corallinus (photo M. R. Duarte).

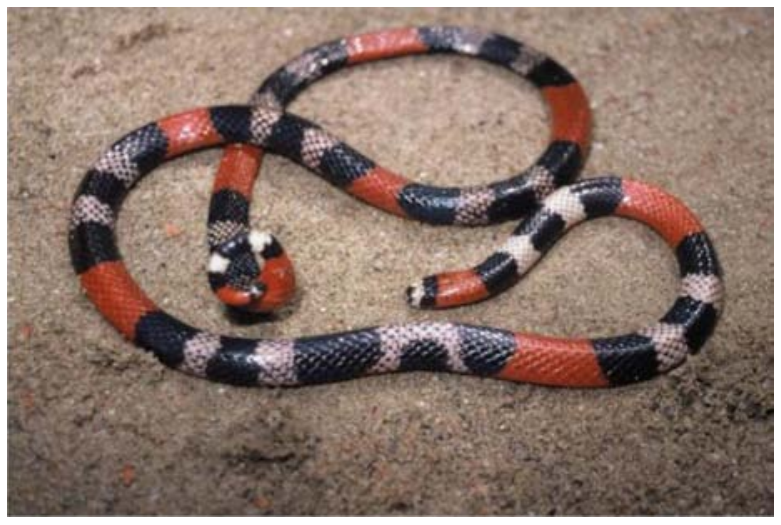

Figura 2. Micrurus ibiboboca (foto M. R. Duarte).

Figure 2. Micrurus ibiboboca (photo M. R. Duarte).
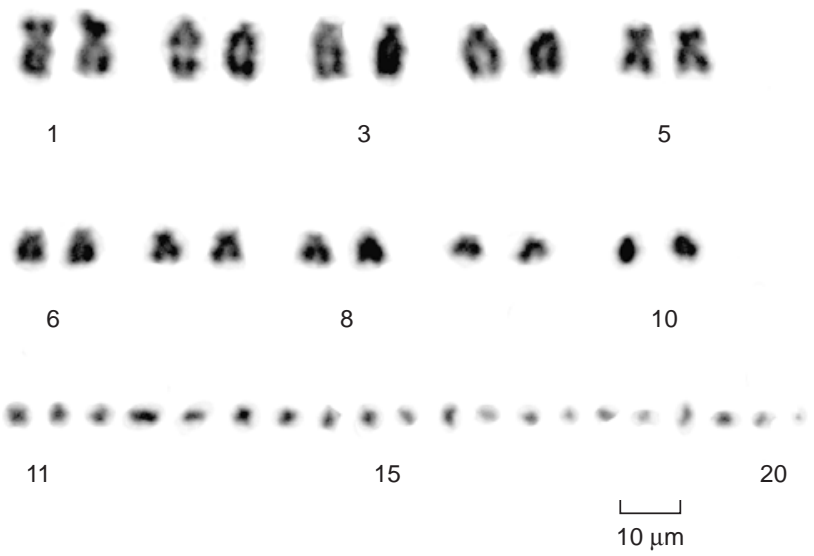

Figura 3. Metáfase mitótica de células epiteliais do intestino de macho de Micrurus corallinus com $2 \mathrm{n}=40$ e fórmula cariotípica $40(4 ; 16 ; 20)$ Note a constrição secundária no segundo par de macrocromossomos subtelocêntricos.

Figure 3. Mitotic metaphase from a Micrurus corallinus intestine epithelial cell with $2 n=40$ and a karyotipic formulae $40(4,16,20)$. Note the secondary constriction in the second pair of a subtelocentric macrochromosome.

Micrurus ibiboboca (Figura 2) apresenta $2 \mathrm{n}=42$ cromossomos, com fórmula cariotípica $42(2 ; 20 ; 20)$. Os macrocromossomos são todos telocêntricos, exceto o par 4 que é submetacêntrico (Figura 4). Os microcromossomos, quando a morfologia pode ser observada, são telocêntricos ou subtelocêntricos. O par 1 apresenta uma constrição secundária (Figura 4).

\section{Discussão}

A análise dos cariótipos descritos neste trabalho mostra diferenças cromossômicas numéricas e estruturais entre as espécies $M$. ibiboboca e M. corallinus. Entretanto, M. ibiboboca e M. corallinus apresentam em comum, 20 microcromossomos, um par de macrocromossomos telocêntricos ou subtelocêntricos com constrição secundária e $\mathrm{NF}=24$. Incluindo os dados deste trabalho, são conhecidos os 
11

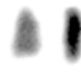

\section{1}

11

3

)

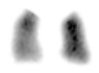

5
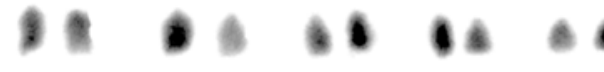

11

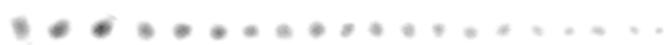

12

21

$\underset{10 \mu \mathrm{m}}{\llcorner}$

Figura 4. Metáfase mitótica de células epiteliais do intestino de macho de Micrurus ibiboboca com $2 \mathrm{n}=42$ e fórmula cariotípica $42(2,20,20)$ Note a constrição secundária no primeiro par de macrocromossomos telocêntricos.

Figure 4. Mitotic metaphase from a Micrurus ibiboboca intestine epithelial cell with $2 n=42$ and a karyotipic formulae $42(2,20,20)$. Note the secondary constriction in the second pair of a subtelocentric macrochromosome.

cariótipos de 13 espécies do gênero Micrurus (Tabela 2). Variações cromossômicas numéricas e estruturais também estão presentes nas demais espécies do gênero. Há uma variação com relação à posição e morfologia do par de cromossomos portador da constrição secundária, tanto nas duas espécies estudadas quanto nas demais descritas (Tabela 2). A maioria das espécies possui constrição secundária no primeiro par de macrocromossomos submetacêntricos. Do ponto de vista citogenético, as espécies da América do Sul, M. lemniscatus (Beçak \& Beçak 1969), M. surinamensis (Gutiérrez \& Bolaños 1988) e as espécies $M$. ibiboboca e $M$. corallinus apresentam semelhanças entre si, que as diferenciam das outras do gênero da América Central.

A partir da Tabela 2 observamos que as espécies da América do Sul apresentam os maiores valores diplóides e de microcromossomos (20), aliados aos mais altos números de macrocromossomos telocêntricos ou subtelocêntricos. Micrurus lemniscatus e M. ibiboboca têm 20 macrocromossomos telocêntricos e subtelocêntricos, M. corallinus, 16 e M. surinamensis, 12 . Quanto ao número diplóide, M. lemniscatus e $M$. ibiboboca têm 42 cromossomos, $M$. corallinus 40 cromossomos e $M$. surinamensis 38 cromossomos. Contudo, todas têm NF $=24$. As espécies da América Central com cariótipos publicados têm todas $\mathrm{NF}=26$. Micrurus alleni apresenta $\mathrm{NF}=26$ se não forem contados os dois pares de macrocromossomos menores, que os próprios autores consideram encontrar-se em uma posição intermediária entre os macrocromossomos e os microcromossomos. Caso estes dois pares sejam contados como macrocromossomos, M. alleni apresentará os maiores números de macrocromossomos entre as espécies não sul-americanas (20), aproximando-se de M. corallinus, $M$. lemniscatus, $M$. ibiboboca e $M$. surinamensis. Considerando-se o número de macrocromossomos telocêntricos e subtelocêntricos e o número de microcromossomos, $M$. alleni apresenta maiores semelhanças com as espécies centro-americanas do que com as sul-americanas (Tabela 2).

Os dados acima mencionados sugerem uma semelhança maior do ponto de vista cariotípico para as espécies da América do Sul. A semelhança é ainda mais nítida entre o grupo de corais de tríades, formado por M. lemniscatus, M. ibiboboca e M. surinamensis. A análise das diferenças cariotípicas que ocorrem entre serpentes do gênero Micrurus da América Central e as da América do Sul, sugere dois padrões evolutivos diferentes. Na América Central, as diferenças de número cromossômico envolvem, principalmente, os microcromossomos e na América do Sul, envolvem os macrocromossomos e o número de microcromossomos permanece constante. Há muita discussão na literatura especializada quanto ao possível número cromossômico ancestral mais provável, não só da Família Elapidae, mas de todas as serpentes. O cariótipo com $2 \mathrm{n}=36$ cromossomos (16 macros + 20 micros) aparece repetidamente em muitas famílias, inclusive as mais antigas como Leptotyphops, Xenopeltis e Boidae (Gorman 1981). Se considerarmos $2 \mathrm{n}=36$ cromossomos um ponto de partida a ser considerado na evolução cromossômica da família Elapidae, outro padrão de evolução cariotípica emerge. As serpentes do gênero Micrurus da América Central tendem a diminuir o número cromossômico, sendo que as de maior número diplóide apresentam $2 \mathrm{n}=34$ e de menor número $2 \mathrm{n}=26$. As espécies da América do Sul tendem a aumentar o número diplóide: $2 \mathrm{n}=38,2 \mathrm{n}=40$ e $2 \mathrm{n}=42$ (3 espécies estudadas). Sugerimos que nas espécies, M. corallinus, M. ibiboboca e $M$. lemniscatus tenha ocorrido fissão cêntrica em macrocromossomos, além de inversões pericêntricas e translocações recíprocas, o que resultou em cariótipos com maior número diplóide e de macrocromossomos telocêntricos e subtelocêntricos.

Nas espécies da América do Sul a constrição secundária aparece no $1^{\circ}$ par de cromossomos telocêntricos ou subtelocêntricos em $M$. ibiboboca e $M$. lemniscatus, enquanto $M$. corallinus tem a constrição no $2^{\circ}$ par de subtelocêntricos e $M$ surinamensis no $2^{\circ}$ par de submetacêntricos. Nas espécies do gênero Micrurus da América Central, a constrição secundária está quase sempre no braço longo do $1^{\circ}$ par de cromossomos, que é metacêntrico ou submetacêntrico. Há exceções em $M$. alleni com par telocêntrico e em M. multifasciatus (tratada por M. mipartitus por Gutiérrez e Bolaños 1979), onde é encontrada no braço longo do $2^{\circ}$ par submetacêntrico. Esta mudança de posição, porém, não reflete necessariamente uma mudança de posição do sítio do gene ribossômico. Pode ser apenas o resultado de perda ou translocação de parte do braço do cromossomo do par 1, formando cromossomos telocêntricos. Por outro lado, pode ser decorrente de perda parcial ou translocação de fragmentos menores, com diminuição do tamanho do cromossomo, que então passaria a ocupar a posição do segundo par. Esta hipótese baseia-se no fato de que quase todas as serpentes do gênero Micrurus centro-americanas apresentam constrição secundária no braço longo do primeiro par.

A partir dos dados de cariótipo conhecidos para o gênero Micrurus, é possível verificar uma correspondência com a análise filogenética proposta por Slowinski (1995), para as cobras corais das Américas (Família Elapidae: Leptomicrurus, Micrurus e Micruroides). A filogenia foi estabelecida através de análise de parcimônia de caracteres morfológicos e alozímicos. Foram estudadas 18 espécies de cobras corais, sendo que outras duas espécies, Bungarus multicinctus e $B$. fasciatus formaram o grupo externo. Posteriormente o autor incluiu outros caracteres morfológicos, acrescentando então mais espécies aos clados formados pela análise de parcimônia.

Das treze espécies do gênero Micrurus que têm seu cariótipo conhecido, nove estão na filogenia proposta por Slowinski (1995). Os dados cariotípicos descritos corroboram em boa parte os grupos propostos por Slowinski (1995). A Figura 5 foi adaptada deste trabalho de Slowinski (1995) e acrescida dos dados cariotípicos descritos para o gênero Micrurus. A partir desta figura pode-se perceber que a filogenia aproximou o grupo de corais de tríades, formado por M. ibiboboca, M. lemniscatus e M. surinamensis. Este grupo é, também, corroborado pelos dados do número diplóide de cromossomos, que são altos, por terem 20 microcromossomos (maiores valores no gênero) e por terem números elevados de macrocromossomos telocêntricos e subtelocêntricos (Tabela 2). Slowinski (1995), após incluir outros caracteres morfológicos ao estudo, sugeriu que 


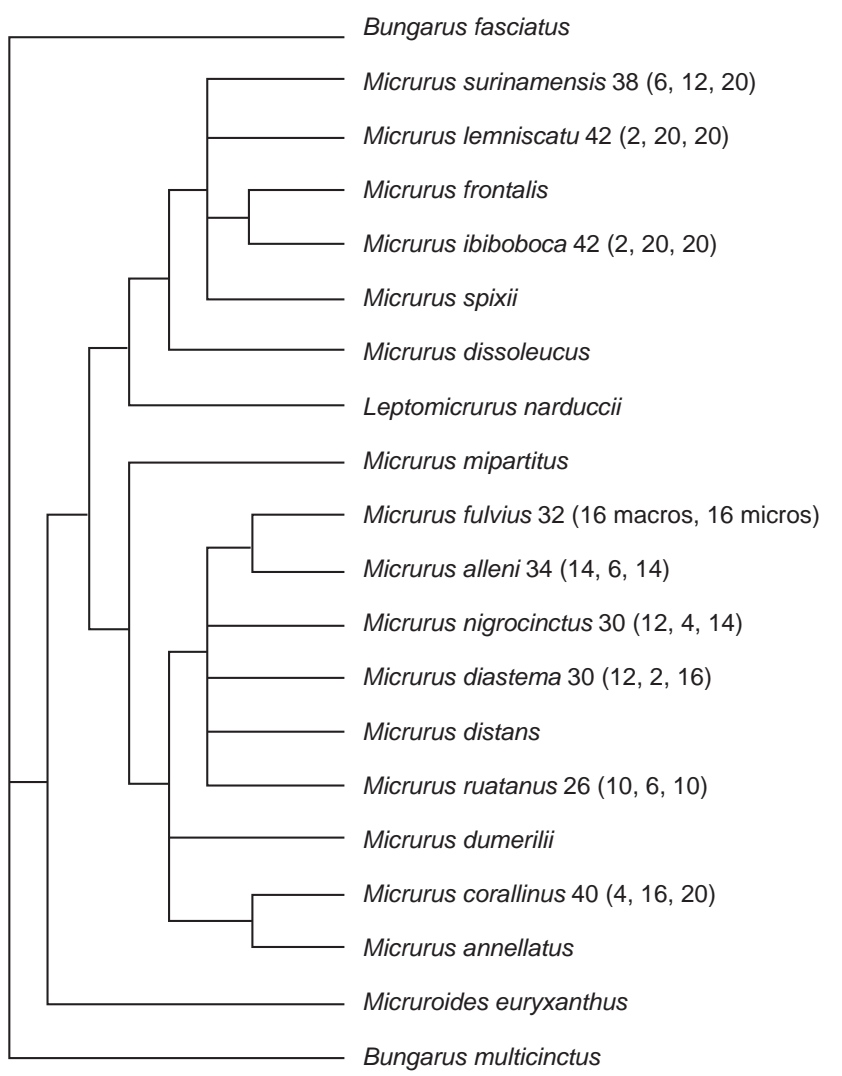

Figura 5. Relações filogenéticas entre algumas espécies do gênero Micrurus com base em caracteres alozímicos e morfológicos (retirado de Slowinski, 1995, p. 333). Ao lado da designação de cada táxon estão indicados os números diplóides segundo Peccinini-Seale (1981) e para M. fulvius estão indicados o número de macros e de micros.

Figure 5. Phylogenetic relationships of some species of the genus Micrurus with morphological and allozyme characters. Adapted from Slowinsky (1995, p. 333). Each taxon has a karyotype description as in Peccinini-Seale (1981). M. fulvius has only the numbers of macro and microchromosomes.

M. surinamensis tem algumas características morfológicas únicas, dentro deste grupo de tríades, o que também ocorre do ponto de vista cariotípico. Micrurus ibiboboca e M. lemniscatus têm 42 cromossomos, 20 macrocromossomos telocêntricos ou subtelocêntricos e uma constrição secundária no primeiro par de cromossomos telocêntricos ou subtelocêntricos, enquanto $M$. surinamensis, tem 38 cromossomos, 12 macrocromossomos telocêntricos ou subtelocêntricos e uma constrição secundária no segundo par de cromossomos submetacêntricos. Na filogenia segundo Slowinski (1995), M. corallinus está posicionada mais próxima do grupo formado por $M$. fulvius, M. alleni, M. diastema, M. nigrocinctus e M. ruatanus (Figura 5). Dentro deste grupo existe uma grande variação no cariótipo, como pode ser observado pela filogenia da Figura 5. Do ponto de vista cariotípico, a espécie $M$. corallinus apresenta maiores semelhanças com o grupo de cobras corais de tríades, $M$. ibiboboca, M. lemniscatus e $M$. surinamensis, se considerarmos: o número diplóide, o número de macrocromossomos telocêntricos ou subtelocêntricos e o número de microcromossomos (20). Os aspectos acima discutidos evidenciam a potencialidade dos estudos citogenéticos para esclarecer as relações filogenéticas no gênero Micrurus. Com este objetivo, estudos com este gênero estão sendo ampliados.

\section{Agradecimentos}

Agradecemos ao Laboratório de Carcinologia, Departamento de Zoologia, Instituto de Biociências, USP, São Paulo, SP, através do Prof. Dr. Carlos Eduardo Falavigna da Rocha, da Dra. Terue Cristina Kihara e do Doutorando Ricardo Lourenço Pinto, pela orientação de uso e utilização do fotomicroscópio óptico digital; à mestranda Susan Ienne da Silva, Laboratório de Biologia molecular de Tripanossomatídeos, Instituto de Química, USP, pela adaptação da filogenia apresentada e pelo auxílio técnico no texto; ao Biólogo Marcelo Ribeiro Duarte, Laboratório de Herpetologia, Instituto Butantan, por fornecer as fotos das serpentes; ao mestrando Marco Aurélio de Sena, Laboratório de Citogenética de Répteis e Artrópodes, Departamento de Genética e Biologia Evolutiva, Instituto de Biociências, USP, pelo auxílio na montagem dos cariótipos; ao técnico de apoio a Pesquisa Valdir José Germano, Laboratório de Herpetologia, Instituto Butantan, pelo auxílio na manipulação dos animais; ao IBAMA/RAN, proc. 02027004018/01-26; a APA Cananéia, Iguape e Peruíbe. Agradecemos ao Programa BIOTA-FAPESP que financiou a pesquisa do projeto Diversidade e Conservação da Fauna de Répteis do Sudeste da Floresta Atlântica, processo n 99/08291-5.

\section{Referências Bibliográficas}

BEÇAK, W. \& BEÇAK, M.L. 1969. Cytotaxonomy and chromosomal evolution in Serpentes. Cytogenetics, 8:247-262.

BOLAÑOS, R. 1982. Serpentes venenosas de Centro America: distribución, características y patrones cariológicos. Mem. Inst. Butantan, 46:275-291.

CADLE, J. \& SARICH, V.M. 1981. An immunological assessment of the phylogenetic position of New World coral snakes. Journal of Zoology, 195:157-167

GORMAN, G. 1981. The chromosomes of Laticauda and a review of karyotypic evolution in the Elapids. Journal of Zoology, 15:225-233.

GRAHAM, G. 1977. The karyotype of the Texas coral snakes, Micrurus fulvius tenere. Herpetologica, 33:345-348.

GUTIERREZ, J.M. \& BOLAÑOS, R. 1979. Cariótipos de las principales serpientes coral (Elapidae:Micrurus) de Costa Rica. Rev. Biol. Trop., 27:57-73.

GUTIERREZ, J.M. \& BOLAÑOS R. 1981. Polimorfismo cromosómico intraespecífico en la serpiente de coral Micrurus nigrocinctus de Costa Rica. Rev. Biol. Trop., 29:115-122.

GUTIERREZ, J.M., SOLORZANO, A. \& CERDAS, L. 1988. Karyotypes of five Species of coral snakes (Micrurus). Journal of Herpetology, 22:109-112

LEVAN, A.; FREDGA, K. \& SANDBERG, A.A. 1964. Nomenclature for centromeric position on chromosomes. Hereditas, 52 (2):201-220.

LUYKX, P. SLOWINSKI, J.B. \& MCCRANIE, J.R. 1992. The karyotype of the coral snake Micrurus ruatanus. Amphibia-Reptilia- E. J. Brill, Leiden, 13:289-292.

MATTHEY, R. 1949. Les chromosomes des Vertébrés. Lausanne. F. Rouge.

MELGAREJO, A.R. 2003. Serpentes peçonhentas do Brasil. In Cardoso, J.L.C.; França, F.O.S., Wen, F. H., Málaque, C.M.S., Haddad Jr., V. (ed). Animais Peçonhentos no Brasil: biologia, clínica e terapêutica dos acidentes. Editora Sarvier, São Paulo.

PECCININI-SEALE, D. 1981. New developments in vertebrate cytotaxonomy. IV. Cytogenetic studies in reptiles. Genetica, 56:123-148.

ROZE, J.A. 1982. New World coral snakes (Elapidae): a taxonomic and biological summary. Mem. Inst. Butantan, 46:305-338.

ROZE, J.A. 1996. Coral Snakes of the Americas: Biology, Identification, and Venoms. Krieger Publishing Company, Florida.

SAVAGE J.M. \& VIAL, J.L. 1973. The venomous coral snakes (genus Micrurus) of Costa Rica. Rev. Biol. Trop., 21:295-349. 
SBH. 2006. Lista de espécies de répteis do Brasil. Sociedade Brasileira de Herpetologia (SBH). Disponível em: http:// www.sbherpetologia.org. br/checklist/repteis.htm.

SILVA, N.J.S.J. \& SITES, J.W.J. 2001. Philogeny of South American Triad Coral Snakes (Elapidae: Micrurus) based on molecular characters. Herpetologica, 57:2-19.

SLOWINSKI, J.B. 1995. A phylogenetic analysis of the New World coral snakes (Elapidae: Leptomicrurus, Micruroides, and Micrurus) based on allozymic and morphological characters. Journal of Herpetology, 29:325-338.
Título: Estudo cariotípico de duas espécies brasileiras do gênero Micrurus (Ophidia: Elapidae).

Autores: Serafim H, Peccinini-Seale, DM e Batistic, RF

Biota Neotropica, Vol.7 (número 1): 2007

http://www.biotaneotropica.org.br/v7n1/pt/abstract?article+ bn01607012007

Recebido em 13/06/06 - Versão reformulada recebida em 20/11/06 - Publicado em 15/01/07

ISSN 1676-0603 